\title{
Enfermedad trofoblástica gestacional en un centro oncológico de Caldas - Colombia durante el periodo $2001-2014$
}

\author{
Carlos Raúl Villegas-Mejía* \\ José Arnoby Chacón-Cardona** \\ Manuel Villegas-Jaramillo***
}

\begin{abstract}
* Médico. Cirujano Oncólogo Clínico y Radioterapeuta. Oncólogos del Occidente SA. Manizales. Caldas. Colombia.
** Médico. Magister en Salud Pública. Profesor Titular. Facultad de Ciencias para la Salud. Departamento Clínico. Universidad de Caldas. Manizales. Caldas. Colombia.

*** Estudiante V Nivel de Medicina. Escuela de Medicina. Facultad de Salud. Universidad de Manizales. Manizales. Caldas. Colombia.

Correspondencia: Dr. Carlos Raúl Villegas Mejía. Oncólogos del Occidente SA. Departamento de Oncología Manizales. Caldas. Colombia. Correo electrónico: caravim@hotmail.com
\end{abstract}

\section{RESUMEN}

Introducción: la enfermedad trofoblástica gestacional representa un espectro de enfermedades con un denominador común que sirve de marcador diagnóstico, control y pronóstico, la Subunidad Beta Gonadotrofina Coriónica Humana. Se pretende revisar el patrón clínico de la enfermedad trofoblástica gestacional en un centro oncológico de tercer nivel. Materiales y Métodos: se constituyó una cohorte retrospectiva de pacientes remitidas a la institución en la sede del departamento de Caldas entre enero de 2001 a diciembre de 2014 , con diagnóstico de enfermedad trofoblástica gestacional, en la cual se analizaron variables de tratamiento y evolución de la enfermedad. Resultados: se obtuvieron 25 pacientes con edad media de 30 años; $68 \%$ tenían síntomas previos al diagnóstico de menos de tres meses; $84 \%$ se diagnosticaron por ecografía y $80 \%$ por legrado; $52 \%$ con mola persistente y $24 \%$ con coriocarcinoma; $80 \%$ en etapa clínica I, $8 \%$ en etapa III y $8 \%$ en etapa IV con metástasis en $36 \%$ al pulmón, $18 \%$ a sistema nervioso central y $9 \%$ a hígado; $76 \%$ clasificadas como bajo riesgo. la media de seguimiento entre diagnóstico y remisión fue 6,3 meses, entre cirugía y remisión 6,4 meses y entre la primera quimioterapia y la remisión 2,9 meses. Sobrevida global de 92\%. Conclusión: los factores de mayor importancia en la ocurrencia y evolución de la enfermedad trofoblástica gestacional son el nivel de Gonadotrofina Coriónica Humana, la histología, la presencia o no de enfermedad metastásica, el uso de multiagentes en quimioterapia y finalmente el índice pronóstico internacional. MÉD.UIS. 2017;30(3):39-49.

Palabras clave: Enfermedad Trofoblástica Gestacional. Epidemiología. Tratamiento. Sobrevida.

\section{Gestational trophoblastic disease in oncological center on Caldas - Colombia during 2001 $-2014$}

\section{ABSTRACT}

Introduction: The gestational trophoblastic disease represents a spectrum of diseases with a common denominator that serves as a marker for diagnosis, monitoring, prognoses and follow-up, the Subunit Beta Human Chorionic Gonadotropin. We review the behavior of gestational trophoblastic disease in a cancer center of third level. Materials and Methods: A retrospective cohort of patients referred to the institution on Caldas department from january 2001 to december 2014, with a diagnosis of gestational trophoblastic disease for analyzing treatment and evolution variables. Results: from 25 patients with median age of 30 years; $68 \%$ had symptoms for less than three months; $84 \%$ are diagnosed by ultrasound and $80 \%$ by curettage; $52 \%$ with persistent mole and $24 \%$ with choriocarcinoma; $80 \%$ clinical stage I, stage III $8 \%$ and $8 \%$ in stage IV with metastatic to lung 36\%, $18 \%$ to central nervous system and $9 \%$ to liver; $76 \%$ classified as low risk; mean follow-up between diagnosis and remission was 6.3 months, between surgery and remission of 6.4 months and between the first chemotherapy and remission 2.9 months. The overall survival was $92 \%$. Conclusion: The factors of greater importance in the occurrence and evolution of gestational trophoblastic disease are the level of the Subunit Beta Human Chorionic Gonadotropin, histology, presence or non-metastatic disease, the use of multi-agent chemotherapy and finally the international prognostic index. MÉD.UIS. 2017;30(3):39-49.

Keywords: Gestational Trophoblastic Disease. Epidemiology. Treatment. Survival.

Artículo recibido el 31 de Marzo de 2016 y aceptado para publicación el 20 de Mayo de 2017 
¿Cómo citar este artículo?: Villegas-Mejía CR, Chacón-Cardona JA, Villegas-Jaramillo M. Enfermedad trofoblástica gestacional en un centro oncológico de Caldas - Colombia durante el periodo 2001 - 2014. MÉD.UIS. 2017;30(3):39-49.

\section{INTRODUCCIÓN}

La Enfermedad Trofoblástica Gestacional (ETG) representa un espectro de enfermedades definidas como un trastorno de la proliferación de las células trofoblásticas, las cuales constituyen un grupo heterogéneo de lesiones interrelacionadas derivadas del epitelio trofoblástico de la placenta'. En la ETC se detecta la Subunidad Beta de Gonadotropina Coriónica Humana $\left({ }_{\beta} \mathrm{GCH}\right)$ que sirve como marcador diagnóstico, pronóstico, de respuesta y de actividad tumoral relacionada con la progresión, la persistencia y la curación de la enfermedad ${ }^{2}$. Estas enfermedades se encuentran en el grupo de enfermedades raras con una alta quimio-sensibilidad, que pueden curarse incluso en casos de extensa diseminación ${ }^{3}$.

Según la Organización Mundial de la Salud (OMS) la ETG se clasifica en dos grupos, la mola hidatiforme la cual puede ser incompleta o completa, y la Neoplasia Trofoblástica Gestacional (NTG) la cual a su vez se divide en mola persistente o invasiva, el coriocarcinoma, el tumor trofoblástico del sitio placentario, la lesión trofoblástica diversa definida como del lecho placentario exagerado y nódulo del lecho placentario, y las lesiones trofoblásticas no clasificadas ${ }^{4}$.

La mola hidatiforme comprende el 90\% de los casos, no es invasiva y se desarrolla como resultado aberrante en la fertilización que conduce a un proceso proliferativo con diferencias en presentación, hallazgos ecográficos y hallazgos cromosómicos que pueden ser diploides o triploides. La mola completa o clásica no tiene feto o embrión y en el $95 \%$ de los casos muestra un cariotipo 46 XX resultado de la fecundación de un ovocito con núcleo inactivo por un espermatozoide haploide que reduplica sus propios cromosomas sin participación de los cromosomas maternos, y en un $5 \%$ de los casos el ovocito inactivo es fecundado por dos espermatozoides haploides, uno con cromosoma $X$ y el otro $Y$, de origen paterno; mientras que la mola incompleta o parcial contiene feto, embrión o saco gestacional, su cariotipo es triploide y es el resultado de la fertilización de un ovocito con carga genética normal por un espermatozoide duplicado o por dos espermatozoides; puede manifestarse como cariotipo 69 XXY (70\%), 69 XXX (28\%) y $69 \mathrm{XYY}(3 \%)^{5}$.

Las otras categorías de ETG representan el componente maligno de la enfermedad, debido a su potencial invasivo local y sistémico y están agrupadas en su definición como NTG que puede desarrollarse de cualquier evento gestacional previo tipo aborto, embarazo ectópico o embarazo normal.

La incidencia de la ETG fluctúa ampliamente en el mundo desde la más baja de 23 por cada 100000 embarazos en Paraguay, a la más alta en Indonesia de 1299 por 100000 embarazos, con variabilidad en parte dependiente de subregistro, diferencias en criterios de diagnóstico o notificación, entre otros ${ }^{6-8}$. En algunos centros hospitalarios los especímenes de aborto o muestras de legrados de sangrado del primer trimestre no se envían al laboratorio histopatológico, por lo que se deja de diagnosticar un número desconocido de casos de ETG.

Las formas malignas, representadas principalmente por el coriocarcinoma, ocurren con una frecuencia de 1 en 20000 a 1 en 40000 embarazos en Estados Unidos y Europa respectivamente. En Asia, África y América Latina oscila entre 1 en 500 a 1 en 1000 embarazos, con marcadas variaciones regionales. En Latinoamérica se desconoce la frecuencia de la ETG dado que la literatura aporta principalmente reportes de casos y experiencias institucionales. Diferentes estudios han reportado datos sobre la ETG con incidencias que puedan tener relación con las condiciones propias de cada región, lo cual no es ampliamente estudiado, pero pueden ser objeto de investigación $n^{9-13}$.

Dado que en Colombia y en el Eje Cafetero los reportes son escasos, se plantea la necesidad de revisar la experiencia en oncólogos del occidente, un centro de atención terciaria multimodal en oncología con sede en cuatro departamentos de Colombia (Caldas, Risaralda, Quindío y Valle del Cauca) donde se atienden los casos remitidos por hospitales y entidades de atención ginecobstétrica de los municipios de influencia de esta institución, con el objetivo de conocer la frecuencia, forma 
de presentación, factores de riesgo, factores pronósticos, métodos diagnósticos, tratamiento y resultados obtenidos en la ETG, en el período 2001 $-2014$.

Los resultados iniciales de este trabajo fueron presentados en el IV Congreso Sudamericano sobre Enfermedad Trofoblástica Gestacional con el título "Tratamiento y seguimiento de la Neoplasia Trofoblástica Gestacional: experiencia en un Centro de Atención Terciaria de Colombia" en Sao Paulo, Brasil. 4 al 6 de diciembre de 2014.

\section{Materiales y métodos}

Se realizó un estudio analítico de cohorte retrospectiva de pacientes remitidas a Oncólogos del Occidente como centro de referencia de enfermedades oncológicas. Se revisó la base de datos del período comprendido entre enero de 2001 a diciembre de 2014, en la cual se registró la información Institucional de la sede localizada en la ciudad de Manizales, departamento de Caldas. La información se extrajo de la historia clínica de ingreso a la institución y se aplicó un formato individual de captura de información específica en la que se consignaron todos los eventos relacionados.

El criterio principal de inclusión fue el diagnóstico patológico de ETG y se excluyeron diagnósticos poco claros o sin confirmación patológica.

Se analizaron variables demográficas (edad, ocupación, estado civil, municipio de procedencia, departamento, zona de procedencia geográfica, hemoclasificación y nivel de escolaridad); clínicas (síntomas al ingreso, estado funcional tipo Karnofsky, método diagnóstico empleado, número de embarazos, partos y abortos; clasificación según el índice pronóstico internacional, comorbilidades presentadas, edad de inicio de vida sexual, número de compañeros sexuales, presencia de enfermedad metastásica, sitio de metástasis, tiempo de inicio de Quimioterapia (QT) con respecto a la Cirugía (Cx), tipo de QT, cambio de esquema de QT y motivo del cambio, número de ciclos de QT y fechas de inicio de QT, fecha de curación, presencia de recaída y fecha de recaída, tratamiento de rescate y esquema de QT de rescate, tipo de $\mathrm{Cx}$ realizada); patológicas (tipo histológico y hallazgos histológicos en la muestra); paraclínicas (cifra de ${ }_{\beta} \mathrm{GCH}$ previa, durante y en cada ciclo de QT). Todos los tiempos se definieron en meses.
Los pacientes perdidos se definieron como aquellos sin datos actualizados ni posibilidades de ubicación por diferentes medios. Adicionalmente, se agrupó la edad en <20 años, 21-40 y mayor de 41 años, el tiempo de síntomas se agrupó en menos de tres, entre tres y seis, y más de seis meses; la recaída se definió desde el punto de vista clínico y/o paraclínico con base en ecografía, tomografía axial computarizada o nivel de ${ }_{\beta} \mathrm{GCH}$ sérica. Por último, el seguimiento se definió como los meses transcurridos entre el diagnóstico y el cierre del estudio (Diciembre de 2014), la fecha del último control en los perdidos o la fecha de fallecimiento; el estado final se definió, según el último control, en dos categorías mayores: $\operatorname{Vivos}(\mathrm{V})$ y $\operatorname{Muertos}(M)$.

Las variables cualitativas se analizaron mediante proporciones y las cuantitativas con promedios y desviaciones estándar. Para supervivencia se utilizó el método de Kaplan-Meier y test de log-Rank. Se utilizaron los programas Epilnfo 6.04d - Epilnfo ${ }^{\text {TM }}$ Versión 3.5 .4 y SPSS versión $14.5^{\oplus}$.

\section{Resultados}

Se encontraron 25 pacientes con diagnóstico de ETG en el período de estudio. La media de edad fue $30 \pm$ 8,9 años (rango: 17 a 57 años). La media de embarazos de la población estudiada fue de 2 (rango 1 a 8 ), la media de partos fue 1,3 (rango: 1 - 5) y la media de abortos fue de 1,1 (rango: 1 a 4) (Ver Tabla 1a).

El principal método diagnóstico empleado fue el ultrasonido (36\%). Los hallazgos anatomopatológicos más relevantes fueron las vellosidades hidrópicas en (26\%). Para el grupo de NTG se encontró el $74 \%$ en etapa clínica I, 10\% para etapa clínica III por metástasis pulmonar y finalmente 10\% en etapa IV por presentar enfermedad metastásica a los pulmones, sistema nervioso central, hígado, bazo, retroperitoneo e intestino (Ver Tabla 1b).

Según el índice pronóstico internacional de la International Federation of Gynecology and Obstetrics (FIGO, por sus siglas en español), en el grupo general se encontró bajo riesgo en el $76 \%$ (puntaje menor 0 igual a seis) y alto riesgo en el $24 \%$ (puntaje mayor o igual a siete), y en el grupo de NTG se encontró $68 \%$ con bajo riesgo y $32 \%$ con alto riesgo. Entre otros factores de riesgo definidos en la literatura, solo se registró el grupo sanguíneo en el $52 \%$ de las pacientes, de los cuales el $85 \%$ pertenecían al grupo O y $15 \%$ al grupo A (Ver Tabla 1 b). 
Tabla 1a. Características demográficas

\begin{tabular}{|c|c|c|}
\hline ASPECTO & $\mathbf{n}$ & $\%$ \\
\hline \multicolumn{3}{|l|}{ GRUPOS DE EDAD (Años) } \\
\hline $0-20$ & 2 & 8 \\
\hline $21-40$ & 3 & 12 \\
\hline$>41$ & 20 & 80 \\
\hline \multicolumn{3}{|l|}{ ZONA } \\
\hline Urbano & 24 & 96 \\
\hline Rural & 1 & 4 \\
\hline \multicolumn{3}{|l|}{ OCUPACIÓN } \\
\hline Hogar & 17 & 68 \\
\hline Estudiante & 2 & 8 \\
\hline Empleado & 2 & 8 \\
\hline Educador & 1 & 4 \\
\hline Coordinador & 1 & 4 \\
\hline Auxiliar de Quirófano & 1 & 4 \\
\hline Secretaria & 1 & 4 \\
\hline \multicolumn{3}{|l|}{ ESTADO CIVIL } \\
\hline Soltera & 11 & 44 \\
\hline Casada & 9 & 36 \\
\hline Unión Libre & 5 & 20 \\
\hline \multicolumn{3}{|l|}{ MUNICIPIO } \\
\hline Pereira & 8 & 32 \\
\hline Manizales & 5 & 20 \\
\hline Armenia & 3 & 12 \\
\hline Otros & 9 & 36 \\
\hline \multicolumn{3}{|l|}{ DEPARTAMENTO } \\
\hline Risaralda & 9 & 36 \\
\hline Caldas & 7 & 28 \\
\hline Quindío & 6 & 24 \\
\hline Valle & 3 & 12 \\
\hline \multicolumn{3}{|l|}{ NIVEL DE ESCOLARIDAD } \\
\hline Sin estudios & 3 & 12 \\
\hline Primaria incompleta & 11 & 44 \\
\hline Secundaria incompleta & 4 & 16 \\
\hline Secundaria completa & 4 & 16 \\
\hline Universitarios & 3 & 12 \\
\hline
\end{tabular}

Tabla 1b. Características clínicas

\begin{tabular}{|c|c|c|}
\hline ASPECTO & $\mathbf{n}$ & $\%$ \\
\hline \multicolumn{3}{|l|}{ SÍNTOMAS } \\
\hline Sangrado vaginal & 22 & 88 \\
\hline Aumento de tamaño uterino & 6 & 24 \\
\hline Test de embarazo positivo & 6 & 24 \\
\hline Pseudo-embarazo & 5 & 20 \\
\hline Anemia & 3 & 12 \\
\hline Dolor & 3 & 12 \\
\hline Descarga vaginal & 2 & 8 \\
\hline Salida de vesículas por vagina & 2 & 8 \\
\hline Hiperémesis & 1 & 4 \\
\hline Presencia de tumor & 1 & 4 \\
\hline \multicolumn{3}{|c|}{ DURACIÓN DE SÍNTOMAS PREVIOS } \\
\hline$<3$ meses & 17 & 68 \\
\hline 3 a 6 meses & 6 & 24 \\
\hline$>6$ meses & 2 & 8 \\
\hline \multicolumn{3}{|l|}{ DIAGNÓSTICO } \\
\hline $\begin{array}{c}\text { Neoplasia Trofoblástica } \\
\text { Gestacional }\end{array}$ & 19 & 76 \\
\hline Mola Completa o Incompleta & 6 & 24 \\
\hline \multicolumn{3}{|l|}{ DIAGNÓSTICO HISTOLÓGICO } \\
\hline Mola Persistente/Invasiva & 13 & 52 \\
\hline Coriocarcinoma & 6 & 24 \\
\hline Mola Completa & 5 & 20 \\
\hline Mola Parcial & 1 & 4 \\
\hline \multicolumn{3}{|l|}{ MÉTODO DIAGNÓSTICO } \\
\hline Ultrasonido & 21 & 84 \\
\hline Biopsia-Succión-Curetaje & 20 & 80 \\
\hline Nivel de ${ }_{\beta} \mathrm{GCH}$ Sérica & 9 & 36 \\
\hline Cirugía & 4 & 16 \\
\hline Sospecha Clínica & 3 & 12 \\
\hline $\begin{array}{c}\text { Diferencia Altura Útero/ } \\
\text { Gestación }\end{array}$ & 1 & 4 \\
\hline \multicolumn{3}{|l|}{ HALLAZGOS HISTOLÓGICOS } \\
\hline Vellosidades Hidrópicas & 12 & 48 \\
\hline Decidua & 11 & 44 \\
\hline
\end{tabular}




\begin{tabular}{|c|c|c|}
\hline ASPECTO & $\mathbf{n}$ & $\%$ \\
\hline Autolisado & 7 & 28 \\
\hline Restos placentarios & 4 & 16 \\
\hline Hiperplasia endometrial & 1 & 4 \\
\hline Otros & 1 & 4 \\
\hline No definido & 11 & 44 \\
\hline \multicolumn{3}{|c|}{ ORIGEN DEL CORIOCARCINOMA } \\
\hline Embarazo Molar & 4 & 67 \\
\hline Embarazo Normal & 2 & 33 \\
\hline \multicolumn{3}{|l|}{ ETAPA CLÍNICA DE ETG } \\
\hline I & 20 & 80 \\
\hline II & 1 & 4 \\
\hline III & 2 & 8 \\
\hline IV & 2 & 8 \\
\hline \multicolumn{3}{|c|}{ ÍNDICE PRONÓSTICO INTERNACIONAL } \\
\hline Bajo Riesgo $(\leq 6)$ & 19 & 76 \\
\hline Alto Riesgo ( $\geq 7$ ) & 6 & 24 \\
\hline \multicolumn{3}{|l|}{ EMBARAZOS } \\
\hline 0 & 4 & 16 \\
\hline 1 & 7 & 28 \\
\hline 2 & 4 & 16 \\
\hline 3 & 5 & 20 \\
\hline 4 & 3 & 12 \\
\hline 5 & 1 & 4 \\
\hline 8 & 1 & 4 \\
\hline \multicolumn{3}{|l|}{ PARTOS } \\
\hline 0 & 10 & 40 \\
\hline 1 & 7 & 28 \\
\hline 2 & 5 & 20 \\
\hline 3 & 2 & 8 \\
\hline 5 & 1 & 4 \\
\hline \multicolumn{3}{|l|}{ ABORTOS } \\
\hline 0 & 10 & 40 \\
\hline 1 & 7 & 28 \\
\hline 2 & 5 & 20 \\
\hline 3 & 2 & 8 \\
\hline 4 & 1 & 4 \\
\hline \multicolumn{3}{|l|}{ HEMOCLASIFICACIÓN } \\
\hline$A+$ & 1 & 4 \\
\hline A - & 1 & 4 \\
\hline $\mathrm{O}+$ & 9 & 36 \\
\hline O - & 2 & 8 \\
\hline
\end{tabular}

\begin{tabular}{|c|c|c|}
\hline ASPECTO & $\mathbf{n}$ & $\%$ \\
\hline No registrado & 12 & 48 \\
\hline \multicolumn{3}{|c|}{ EDAD DE INICIO DE VIDA SEXUAL } \\
\hline $14-17$ años & 14 & 56 \\
\hline 18 - 20 años & 7 & 28 \\
\hline $22-28$ años & 4 & 16 \\
\hline \multicolumn{3}{|c|}{ NÚMERO DE COMPAÑEROS SEXUALES } \\
\hline 1 & 16 & 64 \\
\hline 2 & 7 & 28 \\
\hline 3 & 1 & 4 \\
\hline 4 & 1 & 4 \\
\hline \multicolumn{3}{|l|}{ SITIO DE METASTÁSIS } \\
\hline Pulmón & 4 & 36.4 \\
\hline Sistema nervioso central & 2 & 18.2 \\
\hline $\begin{array}{l}\text { Otros (bazo, hígado, intestino, } \\
\text { retroperitoneo) }\end{array}$ & c/u: 1 & c/u:9.1 \\
\hline
\end{tabular}

Fuente: Base de datos Oncólogos del Occidente-Sede Caldas

Tabla 2. Intervención

\begin{tabular}{|c|c|c|}
\hline ASPECTO & $\mathbf{n}$ & $\%$ \\
\hline \multicolumn{2}{|c|}{ ESQUEMA DE QUIMIOTERAPIA } \\
\hline MTX/Leucovorin Ciclo 8 días & 15 & 60 \\
\hline EMA / CO & 3 & 12 \\
\hline MTX 1-5 Diario IV & 1 & 4 \\
\hline MTX Semanal IM & 1 & 4 \\
\hline MTX / Dactinomicin & 1 & 4 \\
\hline Sin Quimioterapia & 4 & 16 \\
\hline
\end{tabular}

PERIODO ENTRE CIRUGÍA Y OUIMIOTERAPIA (Semanas)

\begin{tabular}{|c|c|c|}
\hline O - 1 & 1 & 4 \\
\hline $1-2$ & 5 & 20 \\
\hline$>2$ & 14 & 56 \\
\hline Sin QT ni CX & 5 & 20 \\
\hline
\end{tabular}

QUIMIOTERAPIA DE RESCATE

\begin{tabular}{|c|l|l|}
\hline EMA / CO & 1 & 50 \\
\hline MAC & 1 & 50 \\
\hline
\end{tabular}

CICLOS DE QUIMIOTERAPIA

\begin{tabular}{|c|c|c|}
\hline No OT & 4 & 16 \\
\hline 1 - 3 ciclos & 10 & 40 \\
\hline 4 - 6 ciclos & 10 & 40 \\
\hline 7 o más & 1 & 4 \\
\hline
\end{tabular}




\begin{tabular}{|c|c|c|}
\hline ASPECTO & $\mathbf{n}$ & $\%$ \\
\hline \multicolumn{3}{|l|}{ TRATAMIENTO FINAL } \\
\hline Cx: Succión Curetaje + OT & 15 & 60 \\
\hline Cx: Succión Curetaje & 4 & 16 \\
\hline Cx: Succión Curetaje + QT + QT & 3 & 12 \\
\hline Cx: Succión Curetaje + Cx: HTA + OT & 2 & 8 \\
\hline Cx: Succión Curetaje + Cx: HTA & 1 & 4 \\
\hline \multicolumn{3}{|c|}{$\begin{array}{l}\text { SEGUIMIENTO ENTRE DIAGNÓSTICO Y CURACIÓN } \\
\text { (Meses) }\end{array}$} \\
\hline 0 a 3 & 6 & 24 \\
\hline 3 a 6 & 8 & 32 \\
\hline 6 a 12 & 8 & 32 \\
\hline Más de 12 & 3 & 12 \\
\hline \multicolumn{3}{|c|}{$\begin{array}{l}\text { SEGUIMIENTO ENTRE PRIMERA QUIMIOTERAPIA Y } \\
\text { CURACIÓN (Meses) }\end{array}$} \\
\hline 0 a 3 & 14 & 56 \\
\hline 3 a 6 & 5 & 20 \\
\hline Más de 6 & 2 & 8 \\
\hline Sin Quimioterapia & 4 & 16 \\
\hline \multicolumn{3}{|c|}{$\begin{array}{l}\text { SEGUIMIENTO ENTRE PRIMERA CIRUGÍA Y CURACIÓN } \\
\text { (Meses) }\end{array}$} \\
\hline 0 a 3 & 5 & 20 \\
\hline 3 a 6 & 7 & 28 \\
\hline 6 a 12 & 10 & 40 \\
\hline 12 o más & 2 & 8 \\
\hline Sin Cirugía & 1 & 4 \\
\hline \multicolumn{3}{|l|}{ ESTADO FINAL } \\
\hline Vivos (V) & 23 & 92 \\
\hline Muertos (M) & 2 & 8 \\
\hline
\end{tabular}

\section{Definición de Siglas:}

ETG: Enfermedad Trofoblástica Gestacional

MTX: Metotrexate

EMA/CO: Etopósido - Metotrexate - Actinomicina D Ciclofosfamida - Vincristina

EMA: Etopósido - Metotrexate - Actinomicina D

IV: Intravenoso

IM: Intramuscular

QT: Quimioterapia

Cx: Cirugía

CO: Ciclofosfamida - Vincristina

MAC: Metotrexate - Actinomicina D - Ciclofosfamida

HTA: Histerectomía

Fuente: Base de datos Oncólogos del Occidente-Sede Caldas.

Después del procedimiento quirúrgico, el $84 \%$ recibió QT. En el $5 \%$ de estos casos se inició tratamiento en la primera semana, $24 \%$ entre la primera y segunda semana y el $71 \%$ después de dos semanas de su procedimiento quirúrgico. Entre las pacientes con QT, el 71\% fueron tratadas con esquema de ocho días de Metotrexate - Leucovorin alterno y un 14\% con esquema EMA/CO (curso 1: Etopósido Metotrexate - Dactinomicin - Folinato de Calcio y curso 2: Vincristina - Ciclofosfamida) y el restante $15 \%$ con esquemas basados en Metotrexate (Ver Tabla 2).

La media de ciclos de QT empleados fue de 3,2 ciclos distribuidos en el $48 \%$ con uno a tres ciclos y $52 \%$ con tres a siete ciclos. El tratamiento final de los pacientes consistió en Cx y QT en el $80 \%$, 72\% con procedimiento quirúrgico tipo legrado y $8 \%$ con procedimiento quirúrgico mixto tipo legrado e histerectomía. En el $20 \%$ restante del global solo se realizó tratamiento quirúrgico tipo succión, curetaje e histerectomía en el $4 \%$ y en $16 \%$ solo succión y curetaje.

El patrón de presentación y la evolución del nivel de ${ }_{\beta} \mathrm{GCH}$ mostró un descenso importante en la sexta medición para las 25 pacientes con una media al inicio de 181 302,52 $\pm 283 \mathrm{UI}$, (rango: $308-1328$ 000) (Ver Gráfica 1).

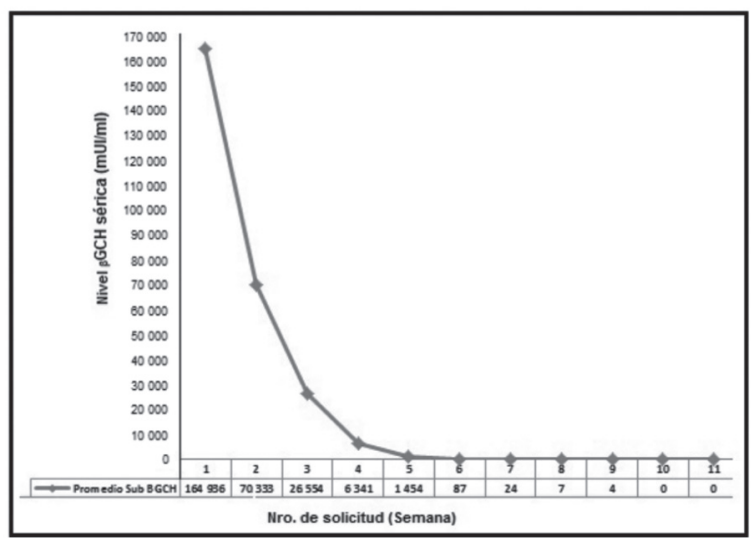

Gráfica 1. Evolución del Promedio general de la Subunidad $\beta G C H$ sérica

Comportamiento del promedio de las 25 pacientes en la muestra general de la Subunidad $\beta \mathrm{GCH}$ sérica desde el momento del diagnóstico hasta el final del estudio.

Sub BGCH: Subunidad ${ }_{\beta}$ GCH

Fuente: Base de datos Oncólogos del Occidente-Seccional Caldas.

Sistema SPSS 11.5 for Win (www.spss.com).

La media de seguimiento global fue de $95,5 \pm 53$ meses (rango: 1 a 194) y la media del período libre de 
enfermedad fue de 89,6 \pm 52,6 meses (rango: 1 a 200)

(Ver Tabla 3).

Tabla 3. Diferencias en el seguimiento de pacientes según intervención (días)

\begin{tabular}{|l|c|c|}
\hline \multicolumn{1}{|c|}{ Aspecto / Tiempo } & Media & $\begin{array}{c}\text { DS } \\
\text { (días) }\end{array}$ \\
\hline Entre diagnóstico y cirugía & 6,15 & 97,38 \\
\hline Entre diagnóstico y quimioterapia & 120,33 & 115,27 \\
\hline Entre cirugía y quimioterapia & 114,15 & 104,11 \\
\hline Entre remisión completa y cirugía & 191,67 & 109,22 \\
\hline $\begin{array}{l}\text { Entre remisión completa y } \\
\text { diagnóstico }\end{array}$ & 189,32 & 122,35 \\
\hline $\begin{array}{l}\text { Entre remisión completa y } \\
\text { quimioterapia }\end{array}$ & 69 & 49,58 \\
\hline
\end{tabular}

DS: Desviación estándar

Fuente: Base de datos Oncólogos del Occidente-Sede Caldas.

La sobrevida global fue del $92 \%$ a 5, 10 y 15 años para el grupo completo de ETG y del 90\% para el grupo de NTG en los mismos periodos analizados. La curación en las pacientes en las etapas clínicas I, II y III fue del $100 \%$, observación hecha a 5, 10 y 15 años. Las dos pacientes que estaban en etapa IV fallecieron por causa de enfermedad metastásica como se mencionó previamente de tipo multiorgánica (pulmón, hígado, vagina, carcinomatosis intestinal, bazo y sistema nervioso central) y de sistema nervioso central (Ver Gráfica 2).

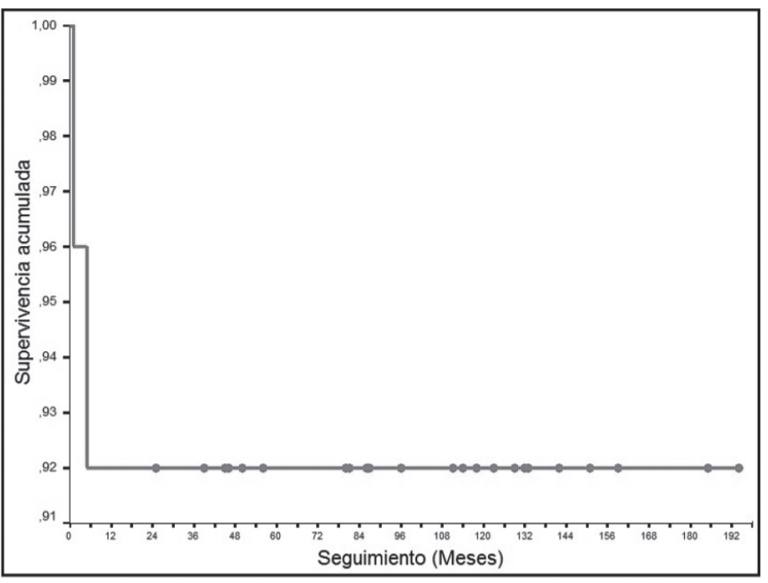

Gráfica 2. Curva de Sobrevida global:

Comportamiento de la curva de sobrevida global de los pacientes Fuente: Base de datos Oncólogos del Occidente-Sede Caldas. Sistema SPSS 11.5 for Win (www.spss.com).

\section{Discusión}

El subtipo molar se presentó similar a lo reportado por otros autores donde la presentación de la NTC se encuentra entre el $60-80 \%$ de los casos; en este trabajo se dio en el $76 \%$ y el $24 \%$ para el tipo molar siendo el mayor porcentaje para la mola persistente en el $52 \%$ similar a otros datos reportados donde la mola persistente o invasiva ocupa el mayor porcentaje $^{8}$

Autores latinoamericanos coinciden que el riesgo de un embarazo molar aumenta por encima de los 40 años, pero considera el grupo etario por debajo de los 20 años, como de alto riesgo ${ }^{8}$. Igualmente, en la literatura se ha sugerido una relación directa con el nivel socioeconómico bajo y la mala alimentación sin embargo, esto no ha sido definido en estudios previos, hasta la fecha hay poca evidencia para sostener esta relación en poblaciones con un nivel de salud y económico más alto como en el Reino Unido y en Hawái. Esta variabilidad podría relacionarse con diferencias genéticas, socioeconómicas, poblacionales, culturales o ambientales, aspectos que no fueron evaluaron en este estudio ${ }^{14}$.

Algunos autores han analizado aspectos como la asociación de la paridad con la incidencia de mola, pero en la serie estudiada no se consideró este aspecto. Otros estudios han analizado aspectos como gravidez, abortos, edad de inicio de relaciones sexuales y número de compañeros sexuales que han sido considerados como factores de riesgo ${ }^{15}$.

Se ha considerado que el grupo sanguíneo A presenta un riesgo mayor de enfermedad contrario al grupo $O$ donde la frecuencia de coriocarcinoma es menor; igualmente se ha definido que las pacientes con grupo $A$ o $A B$ presentan peor pronóstico, pero dado que solo se contaron con datos en el $52 \%$ de los casos y de estos solo se presentaron dos pacientes con subtipo $A$, no es posible coincidir de manera valida este aspecto ${ }^{14}$.

Según el estudio de Goldstein y Berkowitz, la hemorragia vaginal fue la principal manifestación, reportada en el $97 \%$ de las pacientes, lo que concuerda con los resultados presentados ${ }^{16,17}$. La 
presencia de sangrado vaginal, útero agrandado y molestias pélvicas, sumadas a una concentración elevada de ${ }_{\beta} \mathrm{GCH}$ sérica, son las manifestaciones clínicas más frecuentes de una complicación del embarazo, ya sea amenaza, pérdida, aborto o embarazo ectópico, y no la ETG como primera instancia de trabajo en un servicio de urgencias por lo que se constituye como tal en una muy importante opción diagnóstica que debe ser tenida en cuenta ante cualquier alteración de este tipo en una persona con sospecha o confirmación de embarazo, especialmente en el primer trimestre del embarazo ${ }^{14,15}$.

El ultrasonido se ha convertido en la principal ayuda en la detección de anormalidades relacionadas al inicio de la gestación y, aunque es un método operador dependiente, su uso se ha incrementado en los centros obstétricos. La evaluación rutinaria por ultrasonido pre-evacuación para la detección de mola hidatiforme tiene sensibilidad de $44 \%$ y especificidad de $74 \% \%^{18,19}$. Los hallazgos anatomo-patológicos de los especímenes revisados concuerdan con otras series donde prevalece la presencia de vellosidades hidrópicas, la decidua y el autolisado los cuales se presentaban en mayor forma en los especímenes quirúrgicos ${ }^{20}$.

En forma general, la NTG se diagnostica cuando hay evidencia clínica, radiológica, patológica u hormonal de ETG persistente o recidivante según la definición internacional de la FIGO y de la clasificación de la OMS. Con mayor frecuencia el diagnóstico se hace después de un embarazo molar, pero puede ocurrir después de cualquier tipo de gestación tipo embarazo normal u aborto como es referido por la mayoría de autores ${ }^{21}$.

Se encontró enfermedad localizada en el $84 \%$ lo cual no concuerda con la presentación definida por otros autores quienes encontraron que el $30 \%$ de los pacientes desde el inicio se presentaban con enfermedad avanzada o metastásica (etapas III y IV), siendo en su mayor parte a los pulmones, seguido del compromiso vaginal y en menor proporción el compromiso hepático y cerebral22.

Aunque la presencia de enfermedad metastásica varía ampliamente en el momento de la presentación según el tipo de enfermedad analizada. En el grupo de pacientes con NTG (etapa III-IV) se encontró compromiso pulmonar en $36 \%$, sistema nervioso central en $18 \%$ y $9 \%$ para bazo, hígado, intestinal, retroperitoneo y vagina, similar al compromiso orgánico reportado por Goldstein y Berkowitz ${ }^{23}$ donde describe igual compromiso metastásico topográfico pero con diferencia en los porcentajes de presentación para cada sitio (pulmón $80 \%$, vagina $30 \%$, hígado, sistema nervioso central $10 \%$, como principales sitios).

La clasificación según el índice de riesgo pronóstico internacional de la FIGO refleja la respuesta a monoquimioterapia frente a poliquimioterapia en pacientes de bajo o alto riesgo respectivamente. Al aplicar este indicador pronóstico al grupo general estudiado de ETG se encontró que tres cuartas partes del grupo correspondían al grupo de bajo riesgo (76\%), al hacer la subdivisión se encontró que el $52 \%$ en las pacientes con NTG correspondían al grupo de bajo riesgo ( $8 \%$ en coriocarcinoma y $44 \%$ a mola persistente) siendo el $24 \%$ para el subgrupo de mola completa (20\%) e incompleta (4\%) similar a otras series mayores donde el $70 \%$ al $80 \%$, lo constituye el grupo de bajo riesgo en ambas clasificaciones, constituyéndose en un factor pronostico ${ }^{24}$.

La QT es curativa en la mayoría de casos de ETG puesto que estos tumores trofoblásticos malignos son altamente quimio-sensibles y representan uno de los pocos tipos de cáncer para los cuales la terapia de agente único está aún en uso y es considerada altamente efectiva ${ }^{25,26}$.

La elección del esquema de agente único o en combinación, depende de la categoría de riesgo según el índice de riesgo internacional, del estadio de la enfermedad y del empleo previo de medicamentos en las pacientes. En el grupo tratado se empleó QT como tratamiento básico siendo el esquema más usado el Metotrexate / leucovorin seguido de EMA/ $\mathrm{CO}^{27,28}$, otras pacientes se manejaron con esquemas basados en Metotrexate, lo cual concuerda con otros estudios en que los tratamientos giran en torno a este medicamento en forma única o en combinación o simplemente con el modo de aplicación y la frecuencia del mismo, donde el empleo de protocolos con agente único ha logrado remisiones superiores al $90 \%$ en etapa I y al $80 \%$ en etapas II y III concordante con los resultados obtenidos en el presente estudio ${ }^{29-32}$.

El número de ciclos de QT empleados tuvo una media de 3,2 para lograr la remisión, lo cual implica de seis a siete semanas de tratamiento, que concuerda con otros autores quienes demuestran que la respuesta 
es rápida una vez se inicia la aplicación de QT con promedio de uno a dos meses de uso ${ }^{33}$.

La aplicación de tratamiento sistémico con QT en la primera semana luego del procedimiento quirúrgico ha sido estudiada como factor pronóstico y de respuesta, presentando resultados no concluyentes ${ }^{34}$. En la muestra, el 4\% recibió QT en la primera semana y el $56 \%$ después de dos semanas (promedio de 1,2 semanas). Otros autores han considerado la opción de aplazar el inicio de quimioterapia a 24 semanas, sin comprometer los resultados finales, lo cual difiere del concepto previo. En el grupo estudiado la persistencia de enfermedad activa luego de un primer esquema de QT se rescató con base en esquemas de segunda línea tipo EMA/CO y MAC (Metotrexate, Actinomicina D y Ciclofosfamida o Clorambucil) similar a lo definido en la literatura ${ }^{35}$.

El análisis del seguimiento de los pacientes desde diferentes momentos del proceso de atención muestra diferencias de interés, evidenciando que es una enfermedad altamente quimio-sensible y de respuesta rápida una vez se inicia el tratamiento (Ver Gráfica 3).

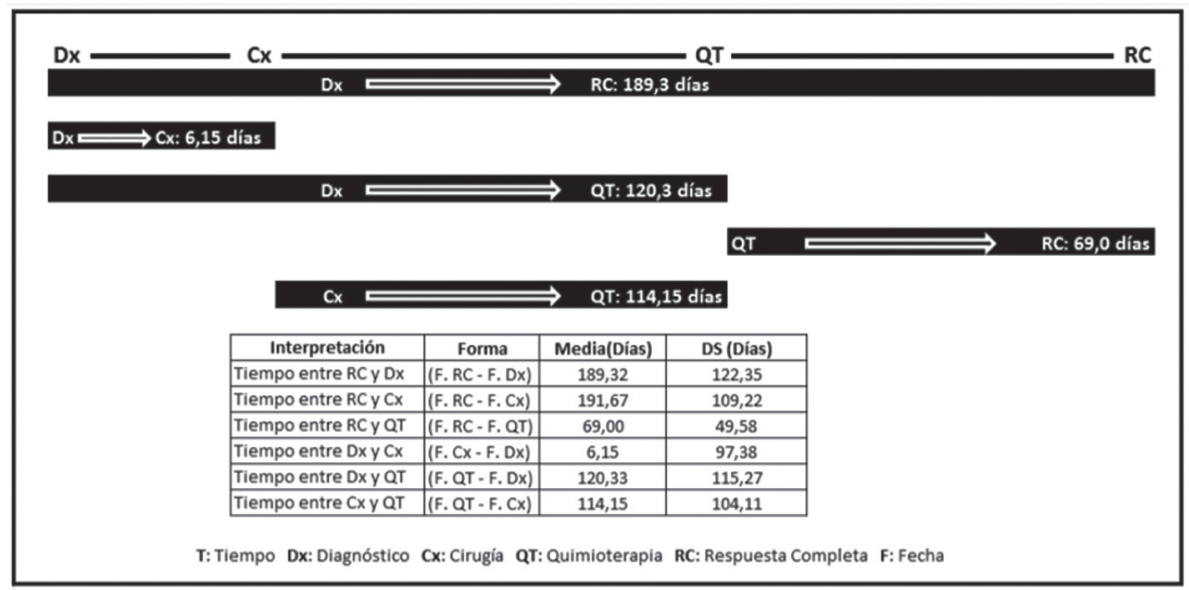

Gráfica 3. Diferencias en días en el seguimiento de los pacientes según la intervención realizada:

Definición según el número de días empleados entre cada una de las intervenciones realizadas para lograr la remisión completa de los pacientes en el tratamiento global

Dx: Diagnóstico, Cx: Cirugía, QT: Quimioterapia, RC: Remisión Completa/Curación, T: Tiempo, F: Fecha, F.RC: Fecha de remisión, F.Dx: Fecha de diagnóstico, F.Cx: Fecha de cirugía, F.OT: Fecha de quimioterapia, DS: Desviación estándar

Fuente: Base de datos Oncologos del Occidente-Sede Caldas.

Sistema SPSS 11.5 for Win (www.spss.com)

La sobrevida global de ETG fue del $92 \%$, la cual puede ser considerada baja ya que en los centros especializados se encuentra cercana al 100\%. Al comparar estudios con similares características, se encuentra el trabajo de Ngan et al. en el cual la sobrevida a 5 años es fue 97,3\% (etapa clínica I), 85,7\% (II) y $82,8 \%$ (etapa clínica III) frente al $92 \%$ para todas estas etapas en Oncólogos del Occidente, pero con una diferencia marcada por los dos pacientes en etapa clínica IV donde se encontró una sobrevida a 5 años de 0\% frente al 61,9\% del estudio de Ngan et al. 35 .

Al analizar la sobrevida con base en la clasificación por índice pronostico internacional se encuentra que el grupo de bajo riesgo presentó a cinco años un $97,3 \%$ en el grupo comparador frente al $100 \%$ en la institución y en el grupo de alto riesgo del 79,5\% en Ngan et al. frente al $67 \%$ en el presente trabajo, con disminución notoria como resultado de los dos pacientes de coriocarcinoma fallecidos 35,36 lo cual podría explicar estas diferencias tan notorias pero sumado al evento anterior también hay que considerar que el número de pacientes es muy pequeño comparativamente a otras series con la salvedad como ya se explicó, de no ser una institución dedicada al tratamiento y estudio de este tipo de patologías lo cual posiblemente estaría en relación a los hallazgos de otros estudios si no se consideran estás variaciones ${ }^{37}$.

Es de anotar que no se encuentran estudios sobre la supervivencia de pacientes con esta patología en Colombia, por lo que se considera de gran importancia continuar este tipo de observación en centros de atención especializada, tanto a nivel gineco-obstétrico como oncológico, ya que los estudios presentes se relacionan con los aspectos 
bioquímico, genético, presentación de caso o revisión de tema ${ }^{38-40}$.

\section{LIMITACIONES}

Se debe considerar principalmente el bajo número de pacientes en el estudio ya que se trata de una institución de tercer nivel de atención oncológica en la cual se observa una frecuencia baja por no ser un centro de atención obstétrica sino de patología oncológica general además de ser un centro de referencia de estudio, diagnóstico y tratamiento tanto de complicaciones oncológicas de otros como de cada una de las neoplasias y como segundo punto importante considerar que se trata de un estudio de carácter retrospectivo.

\section{ConClusión}

Los resultados permiten concluir que el mayor grupo de pacientes estaba en las edades de alto riesgo, con un aumento del tamaño del útero y la presencia de sangrado vaginal anormal entre otros, con síntomas que llevan a consulta rápida, en mayor porcentaje por patología maligna sospechada por ultrasonido y confirmada por biopsia-succión-curetaje y hallazgos histológicos similares a otros reportes lo que condicionó un diagnóstico en etapas iniciales y definidos en su mayor parte por el (empleo del índice de riesgo internacional) como de bajo riesgo. Se empleó principalmente el esquema de quimioterapia basado en Metotrexate/leucovorin semanal con inicio de aplicación menor a dos semanas luego del procedimiento quirúrgico, para obtener una respuesta rápida con una cifra de curación alta ligeramente por debajo del promedio de grandes centros a nivel mundial de manejo de esta patología y datos similares a otros estudios desarrollados en otros lugares.

Estos resultados deben animar a otros grupos, especialmente del área gineco-obstétrica, a adelantar estudios que permitan detectar casos en etapas tempranas y reconocer que la ETG existe y debe ser considerada dentro de las causas probables de sangrado del primer trimestre del embarazo. Además, se genera un llamado al manejo adecuado mediante estudio histopatológico de todas las pacientes atendidas por esta causa tendiente a obtener información de los casos a tiempo para así lograr disminuir la mortalidad en una patología considerada potencialmente curable.

\section{Consideraciones Éticas}

Proyecto avalado por el Departamento de investigaciones de la institución quien permitió el uso de su razón social en el texto; además se ajusta a las normas éticas vigentes en términos de la investigación descriptiva en el área clínica (resolución 8430 de 1993 Ministerio de Salud), donde se demuestra que el trabajo no incide en la evolución o identificación de los pacientes de ningún modo y no hay manipulación diagnóstica, terapéutica o de seguimiento sobre los mismos, constituyéndose en un trabajo sin riesgo para la población de estudio. Se siguen los principios éticos de investigación en humanos o animales según la Declaración de Helsinki de 1975 y revisada en el 2000, la financiación se basó en recursos propios de los investigadores por lo que no existieron conflictos de interés de ningún tipo con empresas privadas o no ni otro tipo de relación con alguna entidad en especial, además los autores declaran no tener impedimentos ni conflictos de interés en la recolección, análisis de la información, ni en los resultados y solo es responsabilidad de los mismos los conceptos allí emitidos.

\section{Financiación}

La totalidad de los gastos generados en revisión de las historias, captura de la información, análisis de los resultados y definición del trabajo como tal corrió por cuenta de los investigadores sin la participación de ninguna entidad externa ni la colaboración de empresas relacionadas.

\section{CONFLICTO DE INTERESES}

No existieron conflictos de interés durante la revisión, análisis o elaboración del manuscrito para ninguno de los investigadores implicados en el trabajo.

\section{Agradecimientos}

A los funcionarios de Oncólogos del Occidente S.A. de las diferentes sedes donde se presta atención oncológica a los pacientes del Eje Cafetero Colombiano.

\section{Referencias Biblográficas}

1. Berkowitz RS. Gestational trophoblastic disease: Epidemiology, clinical manifestations and diagnosis. UpToDate. [Internet] 2014 
[Citado 18 Sep 2017]. Disponible en: https://www.uptodate. com/contents/gestational-trophoblastic-neoplasia-epidemiologyclinical-features-diagnosis-staging-and-risk-stratification

2. Berkowitz RS, Golstein DP. The management of molar pregnancy and gestacional trophoblashc tumours. En: Knapp RC, Berkowitz RS, editores. Gynecologic oncology, 2 ed. Nueva York: Mc Graw - Hill; 1992 p 328-38.

3. Horn LC, Bilek K. Histologic classification and staging of gestacional trophoblastic disease. Gen Diagn Pathol 1997;143(23):87-101.

4. Seckl MJ, Seblre NJ, Berkowitz RS. Gestational trophoblastic disease. Lancet 2010;376(9742):717-29.

5. Brinton LA, Bracken MB, Connelly RR. Choriocarcinoma incidence in the United States. Am J Epidemiol. 1986;123(6):1094-100.

6. Altieri A, Franceschi S, Ferlay J, Smith J, La Vecchia C. Epidemiology and etiology of gestational trophoblastic diseases. Lancet Oncol 2003;4(11):670-8.

7. Palmer JR. Advances in the epidemiology of gestational trophoblastic disease. J Reprod Med 1994;39(3):155-62.

8. Suárez RAE, Santana TRM, Pantoja TCO, Pérez ACE, Vázquez MH. Incidencia de enfermedad trofoblástica gestacional detectada por el estudio histopatológico rutinario de los especímenes obtenidos de abortos. Ginecol Obstet Mex. 2008;76(2):81-7.

9. Rodríguez-Frías E, Mendoza R, León F. Enfermedad del trofoblasto en el Hospital Regional Cayetano Heredia de Piura. Acta Médica Peruana 1985;12:35.

10. Gori RM, Lorusso A. Ginecología de Gori. 2da Ed. Buenos Aires: El Ateneo; 2001. 265-74.

11. Rodríguez M, Aulicino M, Bibbo M, Iglesias F. Enfermedad Trofoblástica Gestacional en un servicio hospitalario de la ciudad de Corrientes. Revista de la Sociedad de Obstetricia y Ginecología.1996;150:192

12. Bianconi MI, Otero S, Moscheni O, Alvarez L, Storino C, Jankilevich G. Gestational Trophoblastic Disease, A 21-year Review of the Clinical Experience at an Argentinean Public Hospital. J Reprod Med. 2012;57(7-8):341-9

13. Suárez RAE, Santana TRM, Pantoja TCO, Pérez ACE, Vázquez MH. Incidencia de enfermedad trofoblástica gestacional detectada por el estudio histopatológico rutinario de los especímenes obtenidos de abortos. Ginecol Obstet Mex. 2008;76(2):81-7.

14. Disaia J. Neoplasia Trofoblastica Gestacional. Edit Panamericana. 1989

15. Buckey J. Epidemiología del embarazo molar y del Coriocarcinoma. Clínicas Obst y srinecol. 1984;1:209-35.

16. Berkowitz RS, Goldstein DP. Chorionic tumors. N Engl J Med 1996; 335(23):1740-8.

17. Soto-Wright V, Bernstein M, Goldstein DP, Berkowitz RS. The changing clinical presentation of complete molar pregnancy. Obstet Gynecol 1995; 86(5):775-9.

18. Mangili G, Spannolo D, Valsecchi L, Maggi R. Transvaginal ultrasonography in persistent trophoblastic tumor. Am J. Obstet Gynaecol.1993;169(5):1218-23.

19. Benson CB, Genest DR, Bernstein MR, Soto-Wright V, Goldstein DP, Berkowitz RS. Sonographic appearance of first trimester complete hydatidiform moles. Ultrasound Obstet Gynecol 2000;16(2):188-91.

20. Baergen RN, Kelly T, McGinniss MJ, Jones OW, Benirschke K. Complete hydatidiform mole with a coexistent embryo. Hum Pathol. 1996;27(7):731-4

21. FIGO Oncology Committee. FIGO staging for gestational trophoblastic neoplasia 2000. FIGO Oncology Committee. Int J Gynaecol Obstet. 2002;77(3):285-7.

22. Edge SB, Byrd DR, Compton CC, et al; American Joint Committee on Cancer. Gestational Trophoblastic Tumors. In: AJCC Cancer Staging Manual. 7th ed. Springer. New York 2010. 437.
23. DuBeshter B, Berkowitz RS, Goldstein DP, Cramer DW, Bernstein MR. Metastatic gestational trophoblastic disease: Experience at the New England Trophoblastic Disease Center, 1965-1985. Obstet Gynecol. 1987;69:390.

24. Mortakis AE, Braga CA. "Poor prognosis" metastatic gestational trophoblastic disease: the prognostic significance of the scoring system in predicting chemotherapy failures. Obstet Gynecol. 1990;76(2):272-7.

25. Goldstein DP, Zanten-Przybysz IV, Bernstein MR, Berkowitz RS. Revised FIGO staging system for gestational trophoblastic tumors. Recommendations regarding therapy. J Reprod Med. 1998;43(1):37-43.

26. Homesley HD. Single-agent therapy for nonmetastatic and low-risk gestational trophoblastic disease. J Reprod Med. 1998;43(1):69-74.

27. Deng L, Zhang J, Wu T, Lawrie TA. Combination chemotherapy for primary treatment of high-risk gestational trophoblastic tumour. Cochrane Database Syst Rev, 2013;31(1):CD005196.

28. Foulmann K, Guastalla JP, Caminet N, Trillet-Lenoir V, Raudrant $\mathrm{D}$, Golfier F, et al. What is the best protocol of single-agent methotrexate chemotherapy in nonmetastatic or low-risk metastatic gestational trophoblastic tumors? A review of the evidence. Gynecol Oncol. 2006;102(1):103-6.

29. Alifrangis C, Agarwal R, Short D, Fisher RA, Sebire NJ, Harvey $\mathrm{R}$, et al. EMA/CO for High-Risk Gestational Trophoblastic Neoplasia: Good Outcomes with Induction Low-Dose EtoposideCisplatin and Genetic Analysis. J Clin Oncol. 2013;31(2):280-6

30. Soper JT, Mutch DG, Schink JC; American College of Obstetricians and Gynecologists. Diagnosis and treatment of gestational trophoblastic disease: ACOG Practice Bulletin No. 53. Gynecol Oncol. 2004;93(3):575-85.

31. Lurain JR. Gestational trophoblastic disease II: classification and management of gestational trophoblastic neoplasia. Am J Obstet Gynecol. 2011;204(1):11-8.

32. Lybol C, Westerdijk K, Sweep FC, Ottevanger PB, Massuger LF, Thomas CM. Human chorionic gonadotropin (hCG) regression normograms for patients with high-risk gestational trophoblastic neoplasia treated with EMA/CO (etoposide, methotrexate, actinomycin D, cyclophosphamide and vincristine) chemotherapy. Annals of Oncology. 2012;23(11):2903-6

33. Gillespie AM, Kumar S and Hancock BW. Treatment of persistent trophoblastic disease later than 6 months after diagnosis of molar pregnancy. Br J Cancer. 2000; 82(8):1393-5.

34. Xiang Y, Sun Z, Wan X, Yang X. EMA/EP chemotherapy for chemorefractory gestational trophoblastic tumor. J Reprod Med. 2004;49(6):443-6.

35. Ngan HY, Odicino F, Maisonneuve P, Creasman WT, Beller U, Quinn MA, et al. Gestational trophoblastic neoplasia. FIGO 26th Annual report on the results of treatment in gynecological cancer. Int J Gynaecol Obstet. 2006;95 Suppl 1:S193-203.

36. Maestá I, Growdon WB, Goldstein DP, Bernstein MR, Horowitz NS, Rudge MV, et al. Prognostic factors associated with time to hCG remission in patients with low-risk postmolar gestational trophoblastic neoplasia. Gynecol Oncol. 2013;130(2):312-6.

37. Seckl M, Seribe R, Fisher RA, Golfier F, Massuger L, sessa C, et al. Gestational trophoblastic disease: ESMO Clinical Practice Guidelines for diagnosis, treatment and follow-up. Ann Oncol. 2013;24 Suppl 6:S39-50.

38. Bermúdez AJ, Cortés C, Díaz LE, Crane C, Ching R, Aragón M, et al. Estudio bioquímico y genético de la enfermedad trofoblástica gestacional. Med. 2006;28(1):14-18.

39. Carrillo DC, García CA. Coriocarcinoma metastásico posparto: presentación de un caso. Rev Colomb Radiol. 2013;24(2):3705-8.

40. Oróstegui S, Arenas YA, Galindo LM. Enfermedad trofoblástica gestacional. MedUNAB. 2008;11(2):140-8. 